\title{
Inhibition of Surface Chemical Moieties by Tris(hydroxymethyl)aminomethane: A Key to Understanding Oxygen Reduction on Iron-Nitrogen-Carbon Catalysts
}

\author{
Y. Chen, ${ }^{\dagger}$ K. Artyushkova, ${ }^{* \dagger}{ }^{\dagger}$ S. Rojas-Carbonell, ${ }^{\dagger}$ A. Serov, ${ }^{\dagger}$ I. Matanovic, ${ }^{\dagger} \ddagger$ C. Santoro, ${ }^{\dagger}$ T. Asset, $^{\dagger}$ \\ and P. Atanassov* ${ }^{* \dagger}+$ (i) \\ ${ }^{\dagger}$ Department of Chemical and Biological Engineering, Center for Micro-Engineered Materials (CMEM), University of New Mexico, \\ Albuquerque, New Mexico 87131, United States \\ ${ }^{\ddagger}$ Theoretical Division, Los Alamos National Laboratory, Los Alamos, New Mexico 87545, United States
}

Supporting Information

\begin{abstract}
The effect of tris(hydroxymethyl)aminomethane (Tris) on the activity of metal-nitrogen-carbon (M-N-C) platinum metal group-free (PGM-free) catalysts for oxygen reduction reaction (ORR) is studied. Electrochemical tests performed in the acidic and alkaline electrolyte and comparison between electrochemical observations for metal-free and metal-containing catalysts along with density functional calculations showed the multiplex mechanism of oxygen reduction in which numerous species perform partial and full reduction of oxygen to peroxide or water, respectively. The important role of protons in the mechanism of oxygen reduction is highlighted.
\end{abstract}

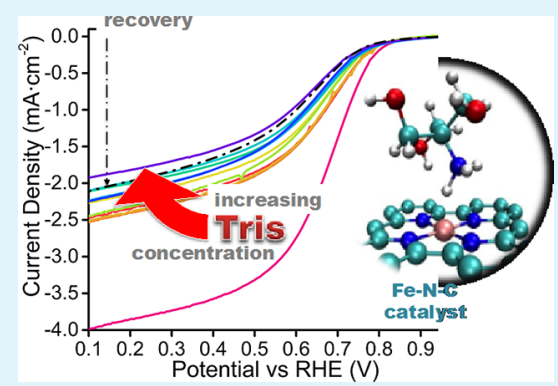

KEYWORDS: platinum group metal-free electrocatalyst, transition metal-nitrogen-carbon catalysts, oxygen reduction reaction, active site poisoning, ORR inhibition, probing active sites

\section{INTRODUCTION}

Metal-nitrogen-carbon $(\mathrm{M}-\mathrm{N}-\mathrm{C})$ materials are among the most studied candidates to replace costly platinum group metal (PGM) catalysts ${ }^{1,2}$ for oxygen reduction reaction (ORR). Numerous methods for $\mathrm{M}-\mathrm{N}-\mathrm{C}$ synthesis proposed in the literature are based on pyrolysis (high-temperature treatment) of macrocycles, ${ }^{1}$ metal-organic frameworks, ${ }^{2}$ and other organic precursors. ${ }^{3}$ To optimize the synthesis of $\mathrm{M}-\mathrm{N}-\mathrm{C}$ structures with best activity and durability, ${ }^{4-6}$ it is essential to understand the mechanism of oxygen reduction reaction and to identify the active sites responsible for different pathways of the ORR. ${ }^{4,6-9}$ Catalyst inhibition or catalyst poisoning are powerful methods to indisputably confirm the role of various metal, carbon, and nitrogen moieties in the individual steps of ORR, such as (1) reduction of oxygen to hydrogen peroxide, (2) the reduction of hydrogen peroxide to water, and (3) the reduction of oxygen directly to water. Some early studies attempted to introduce traditional inhibitors into the electrolyte during oxygen reduction progress, such as carbon monoxide, cyanide, fluoride, thiocyanate, alcohols, thiol, chloride, sulfate, and sulfide. ${ }^{10-15}$ Another approach was based on the introduction of the blocking ligands during the material synthesis, in particular, the hydrogen sulfide-rich atmosphere during heat treatment, ${ }^{10}$ or sulfur, thio-urea, and thiophene mixed with precursors before pyrolysis. ${ }^{16}$ However, these methods have the disadvantage of the excessively strong interaction of these inhibitors with the catalyst resulting in incomplete removal of adsorbed com- pounds, which in turn leads to difficulties in the deconvolution of the active sites in the PGM-free catalysts.

In the latest work focused on the mechanism of ORR through the characterization of inhibited active sites, Kneebone et al. used Mössbauer spectroscopy, nuclear resonance vibrational spectroscopy, and density functional theory to study the influence of $\mathrm{Fe}-\mathrm{N}-\mathrm{C}$ activity toward oxygen reduction under the selective interaction of catalyst active centers with NO. ${ }^{17}$ Mamtani et al. have used phosphate anion to probe the active sites of nitrogen-doped metal-free carbon nanostructures. 8 They observed a correlation between the increase in the level of protonation of the pyridinic sites and decrease of the activity toward oxygen electroreduction. Recently, Malko et al. ${ }^{18}$ studied the poisoning effect of $\mathrm{NO}_{2}{ }^{-}$, $\mathrm{NO}$, and $\mathrm{NH}_{2} \mathrm{OH}$ on the electrocatalytic activity of $\mathrm{Fe}-\mathrm{N}-\mathrm{C}$ catalysts in the acid and alkaline media.

Herein, we report for the first time the use of tris(hydroxylmethyl)-aminomethane (Tris) as a specific molecular probing agent of active sites in $\mathrm{Fe}-\mathrm{N}-\mathrm{C}$ catalysts. Two catalysts, one metal-free $(\mathrm{N}-\mathrm{C})$ and one metal-containing $(\mathrm{Fe}-\mathrm{N}-\mathrm{C})$, were fabricated by identical synthetic procedure from the same carbon-nitrogen precursor, aminoantipyrine (AAPyr), without and with Fe salt (AApyr and FeAApyr, respectively). ${ }^{19-21}$ Study of inhibition and recovery of metal-containing catalysts in the

Received: January 4, 2018

Accepted: April 10, 2018

Published: April 10, 2018 


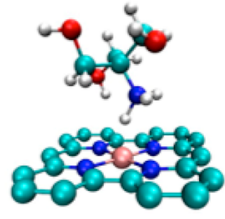

(a)

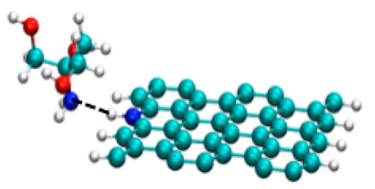

(c)

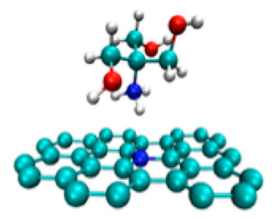

(b)

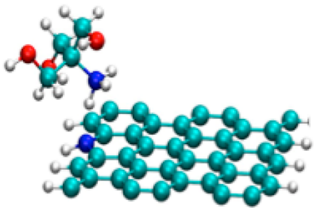

(d)

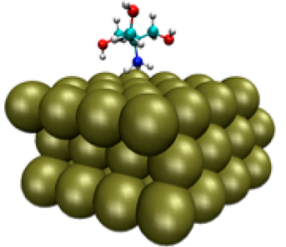

(e)

Figure 1. DFT optimized geometries of TrisH adsorbed on (a) Fe- $\mathrm{N}_{x}$, (b) graphitic N, (c) pyridinic-N, (d) hydrogenated pyridinic-N site, and (e) $\mathrm{Pt}$ (111). For clarity, for $\mathrm{Fe}-\mathrm{N}-\mathrm{C}$ only atoms belonging to part of the unit cell are shown.

acidic and alkaline electrolyte and comparison between metalfree and metal-containing PGM-free catalysts allowed us to correlate the nature of their active sites with individual steps of oxygen reduction reaction.

\section{EXPERIMENTAL SECTION}

Catalysts Preparation. The catalysts used in the presented work were prepared using the sacrificial support method (SSM) as previously presented. ${ }^{14,22}$ Aminoantipyrine (AAPyr) was employed in both cases as a nitrogen-rich organic precursor for the synthesis. FeAAPyr was prepared by mixing iron nitrate and AAPyr. In the case of metal-free catalyst (AAPyr), the only organic precursor was used. In both cases, the monodispersed silica with a surface area of $250 \mathrm{~m}^{2} \mathrm{~g}^{-1}$ was impregnated with mentioned above precursors. The material was then homogenized using ball milling for $30 \mathrm{~min}$. High-temperature treatment was done in a quartz tube in an inert atmosphere of UHP nitrogen. The gas flow was constant at $100 \mathrm{~mL} \mathrm{~min}{ }^{-1}$. The temperature was increased from room temperature to $950{ }^{\circ} \mathrm{C}$ with a ramp rate of $25{ }^{\circ} \mathrm{C} \mathrm{min}^{-1}$. The sample was heat treated for $45 \mathrm{~min}$. The silica template was etched using $20 \mathrm{wt} \% \mathrm{HF}$ overnight $(\sim 12 \mathrm{~h})$. The catalyst was then washed with DI water until the neutral $\mathrm{pH}$ of the supernatant was reached. The catalyst was then dried.

Electrochemical Measurements. RRDE was the technique used to determine the electrocatalytic performances of FeAAPyr and AAPyr in acidic media with/without the addition of Tris. The ink was prepared as previously presented. ${ }^{23,24}$ Five mg of PGM-free catalyst was added to a plastic vial with $1 \mathrm{~mL}$ of $150 \mu \mathrm{L}$ of $0.5 \mathrm{wt} \%$ Nafion solution and $850 \mu \mathrm{L}$ of IPA: $\mathrm{H}_{2} \mathrm{O}$ 1:1 ratio. The mixture was sonicated few times to obtain a uniformly dispersed ink. The latter was drop cast to the disk electrode using a micropipette. The catalyst loading on the disk was $200 \mu \mathrm{g} \mathrm{cm}^{-2}$. The ink was left to dry at room temperature, and then linear sweep voltammetry (LSV) was performed. LSV was run from 0.8 to $-0.2 \mathrm{~V}$ vs $(\mathrm{Ag} / \mathrm{AgCl})$ at a scan rate of $5 \mathrm{mV} \mathrm{s}^{-1}$ for FeAAPyr sample, while from 0.65 to $-0.35 \mathrm{~V}$ for AApyr sample. A graphite rod electrode (PINE research) was used as the counter electrode. After every test, a calculated amount of Tris was added, and the $\mathrm{pH}$ was controlled to be lower than 1. Two series of electrochemical inhibition studies were done independently. The first set of experiments tested electrode made from FeAAPyr catalyst with the following concentrations of Tris in $0.5 \mathrm{M} \mathrm{H}_{2} \mathrm{SO}_{4}$ and in $1 \mathrm{M}$ KOH: $0.001 \mathrm{M}, 0.01 \mathrm{M}, 0.1 \mathrm{M}, 0.8 \mathrm{M}$. The second study tested both FeAApyr and AApyr in $1 \mathrm{M} \mathrm{HClO}_{4}$ using the following concentrations of Tris: $0.1 \mathrm{M}, 0.15 \mathrm{M}, 0.2 \mathrm{M}, 0.25 \mathrm{M}, 0.3 \mathrm{M}, 0.4 \mathrm{M}, 0.5 \mathrm{M}, 0.6 \mathrm{M}, 0.7$ $\mathrm{M}, 0.8 \mathrm{M}$. After LSV was recorded in the $0.8 \mathrm{M}$ solution of Tris, the electrolyte was changed to a freshly prepared $0.5 \mathrm{M} \mathrm{H}_{2} \mathrm{SO}_{4}, 1 \mathrm{M}$ $\mathrm{HClO}_{4}$ or $1 \mathrm{M} \mathrm{KOH}$ while the working electrode was immersed and kept $1600 \mathrm{rpm}$ rotating in deionized (DI) water for $5 \mathrm{~min}$ (DI water was changed 3 times). After washing, the working electrode was used for ORR measurements in the Tris-free electrolytes. The tests under this condition are designated as " $R$ " (recovery or refresh). The reference tests of commercial $\mathrm{Pt}$ were performed in Tris in $0.5 \mathrm{M}$ $\mathrm{H}_{2} \mathrm{SO}_{4}$ using the following concentrations of Tris: $0.001 \mathrm{M}, 0.01 \mathrm{M}$, $0.1 \mathrm{M}, 0.8 \mathrm{M}$. The ink formulation was modified for the commercial $\mathrm{Pt} / \mathrm{C}$ (Aldrich, $10 \mathrm{wt} \%$ of $\mathrm{Pt}$ ), to achieve a $40 \mu \mathrm{g} \mathrm{cm}^{-2}$ loading while maintaining an identical Nafion $(\mathrm{N})$ over Carbon (C) ratio with the PGM-free ink $(\mathrm{N} / \mathrm{C}=$ ca. 0.15$)$.

Computational Details. The calculations were performed using plane-wave pseudopotential implementation of Density Functional Theory (DFT) $)^{25,26}$ with the Perdew-Burke-Ernzerhof (PBE) functional ${ }^{27,28}$ with the use of Vienna Ab initio Simulation Package (VASP). ${ }^{29,30} \mathrm{Fe}-\mathrm{N}_{x}$ and graphitic nitrogen sites were modeled using extended surfaces with the dimensions of $17.04 \AA$ x $17.04 \AA, \gamma=60^{\circ}$ and a vacuum region of $20 \AA$. Pyridinic and hydrogenated pyridinic nitrogen were modeled using the nanoribbons, which were constructed from $4 \times 2$ orthorhombic super cells with the size of $9.84 \AA \times 23.52 \AA$ and with a vacuum regions of $15 \AA$ in the $z$ - and $y$ direction. $\mathrm{Pt}(111)$ surface was modeled using three layers of $\mathrm{Pt}$ atoms in the unit cell of the size $11.2 \times 11.2 \AA$ and a vacuum region of $20 \AA$. The electronic energies were calculated using tetrahedron method with Blöchl correction ${ }^{31}$ and $3 \times 3 \times 1$ k-point Monkhorts-Pack ${ }^{32}$ mesh in the case of the extended surfaces and $8 \times 1 \times 1 \mathrm{k}$-points in the case of the super cells used to model edge defects. In all the cases, plane-wave basis cut off was set to $700 \mathrm{eV}$. Adsorption energies of Tris and protonated Tris (TrisH) at different defects $\left(\Delta E_{\text {ad }}\right)$ were calculated using the following formula:

$$
\Delta E_{\text {ad }}=E_{\text {surface }+ \text { ad }}-\left[E_{\text {surface }}+E_{\text {ad }}\right]
$$

where $E_{\text {surface+ad }}$ is the energy of Tris or TrisH adsorbed on the certain defect, $E_{\text {surafce }}$ is the energy of the extended surface or a nanoribbon, and $E_{\text {ad }}$ is the energy of the Tris/TrisH molecule in the gas phase. In each case, we considered multiple adsorption orientations, but the results reported in work correspond to most preferable adsorption orientation.

\section{RESULTS AND DISCUSSION}

We first used density functional theory (DFT) to understand the interaction of Tris and TrisH with different defects that exist in $\mathrm{Fe}-\mathrm{N}-\mathrm{C}$ materials. At the acidic $\mathrm{pH}$, Tris with $\mathrm{pK}$ value of 8.07 is present in its protonated form, which we denote TrisH, while at alkaline $\mathrm{pH}$ it is present in deprotonated form Tris. The interaction of Tris and TrisH with different defects was calculated as shown in Figure 1. The defects considered in the calculations are responsible for $2 \times 2 \mathrm{e}^{-}$or $4 \mathrm{e}^{-}$mechanism of oxygen reduction to water (metal-nitrogen directly 
coordinated), $2 \mathrm{e}^{-}$reduction of oxygen to peroxide (hydrogenated pyridinic, pyrrolic nitrogen and graphitic nitrogen) and reduction of peroxide to water (pyridinic nitrogen). ${ }^{7,3}$

The DFT results show that the interaction energy between the $\mathrm{Fe}-\mathrm{N}_{4}$ site and Tris of $-0.25 \mathrm{eV}$ is significantly smaller than that of oxygen (see Table 1). Therefore, we assume that

Table 1. Adsorption Energy of Tris, Protonated Tris (TrisH), and Oxygen in $\mathrm{eV}$ as Calculated Using DFT

\begin{tabular}{lllc}
\multicolumn{1}{c}{ site } & \multicolumn{1}{c}{ TrisH } & Tris & \multicolumn{1}{c}{$\mathrm{O}_{2}$} \\
(a) $\mathrm{Fe}-\mathrm{N}_{x}$ & $-1.96(x=4)$ & -0.25 & $-1.01^{a}$ to $-1.43^{b}$ \\
& $-2.18(x=2)$ & $(x=4)$ & $\begin{array}{l}(x=4)-1.68^{a} \\
(x=2)\end{array}$ \\
& & & $-0.13^{a}$ to $-0.41^{b}$ \\
(b) graphitic-N & -1.07 & -0.02 & $-0.08^{a}$ to $-0.28^{b}$ \\
(c) pyridinic-N & -3.65 & -0.41 & $-0.21^{b}$ to $-0.25^{b}$ \\
(d) hydrogenated & -0.53 & -0.26 & \\
pyridinic-N & & & \\
/pyrrolic $\mathrm{N}$ & & & -0.44 to $-0.81^{c}$ \\
(e) Pt $(111)$ & -3.07 & &
\end{tabular}

${ }^{a}$ Values calculated using PBE functional and as published in ref 34 . ${ }^{b}$ Values calculated using vdW-DF functional as published in ref 35 . ${ }^{c}$ Values are taken from refs 36-38.

Tris form does not bind to metal-containing active sites and is not involved in the inhibition process, by opposition to TrisH $(-1.96 \mathrm{eV})$. From the comparison of the adsorption energies of TrisH and $\mathrm{O}_{2}$ on different defects (Table 1), it can be concluded that TrisH has the strongest interaction with pyridinic- $\mathrm{N}$ and $\mathrm{Fe}-\mathrm{N}_{x}$ sites, followed by the graphitic- $\mathrm{N}$ and hydrogenated nitrogen defects (pyrrolic and hydrogenated pyridine). At the same time, only pyridinic nitrogen serves as a possible site for Tris binding that is strong enough to compete with the binding of oxygen. $\mathrm{Fe}-\mathrm{N}_{x}$ and pyridinic nitrogen are the most preferred sites for TrisH binding and for these sites the binding energy of TrisH is significantly larger than that of oxygen.

Electrocatalytic activity of FeAAPyr in the reaction of oxygen reduction was evaluated by Rotating Ring Disk Electrode (RRDE) in the $0.5 \mathrm{M} \mathrm{H}_{2} \mathrm{SO}_{4}$ and $1 \mathrm{M} \mathrm{KOH}$ (Figure 2). The information derived from linear sweep voltammograms (LSVs) as a function of Tris concentration is presented in Figure 3. LSV for commercial $\mathrm{Pt} / \mathrm{C}$ was also acquired as a reference. According to DFT, there is a strong energy of adsorption between $\mathrm{Pt}$ and TrisH $(-3.07 \mathrm{eV}$, see Table 1 and Figure $1 \mathrm{e})$, which is comparable to the energy of adsorption of TrisH to pyridinic N. In Pt/C where ORR occurs via direct $4 \mathrm{e}^{-}$ mechanism, addition of TrisH causes very significant decreases in half-wave potential due to blockage of $\mathrm{Pt}$ sites by the absorbed inhibitor (Figure 3h), while limiting disk current decreases insignificantly at lower concentrations than $0.1 \mathrm{M}$ and drops significantly at $0.8 \mathrm{M}$ of Tris (Figure 2c). This indicates that at larger concentrations of Tris, i.e., above $0.1 \mathrm{M}$, solubility of oxygen in the electrolyte decreases due to the increased concentration of inhibitor. This explanation is further supported by the negligible (and independent of the Tris concentration) ring current (Figure 2f). Indeed, this result confirms that oxygen reduction reaction on Pt follows a direct $4 \mathrm{e}^{-}$pathway and that Tris addition does not have any effect on the hydrogen peroxide yield. Because the limiting current is dependent on the reactant concentration in solution and on the exchanged number of electrons. The constant value of the later confirms that Tris is decreasing $\mathrm{O}_{2}$ solubility in solution at concentrations above $0.1 \mathrm{M}$. Washing away Tris from the working $\mathrm{Pt} / \mathrm{C}$ electrode results in full recovery of both halfwave potential and disk current at $0.2 \mathrm{~V}$.

For FeAAPyr catalyst, the addition of even $0.001 \mathrm{M}$ Tris to electrolyte results in $25 \mathrm{mV}$ shift of half-wave potential to a lower value in acidic solution and no change in alkaline. In
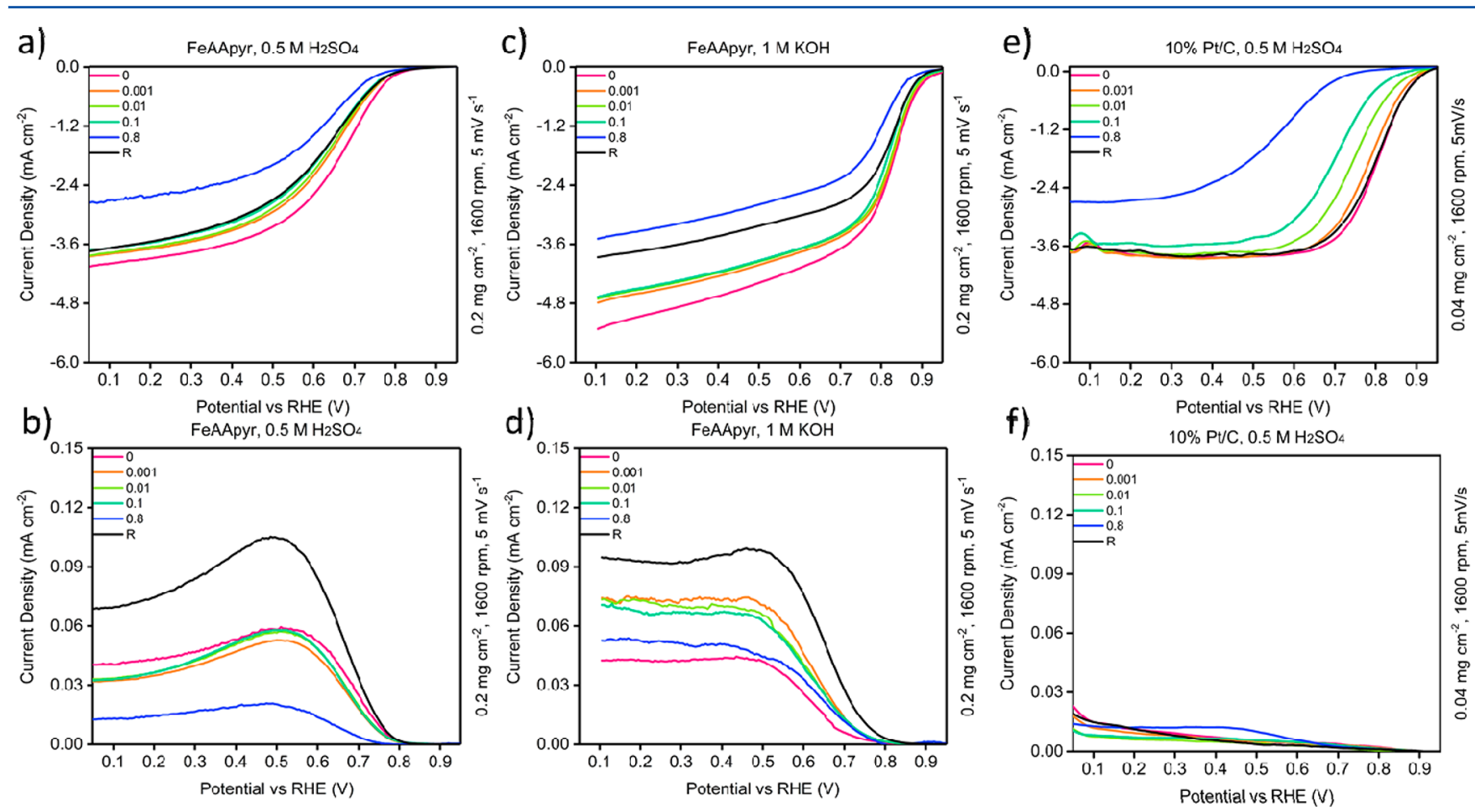

Figure 2. LSV on (a, c, e) disk electrode and $(b, d, f)$ ring electrode in different concentrations of Tris, where " $R$ " denotes refreshed Tris-free electrolyte and washed RRDE. Data of (a, b) FeAApyr in acid, (c, d) FeAApyr in alkaline, and (e, f) Pt/C in acid, respectively. 
a)

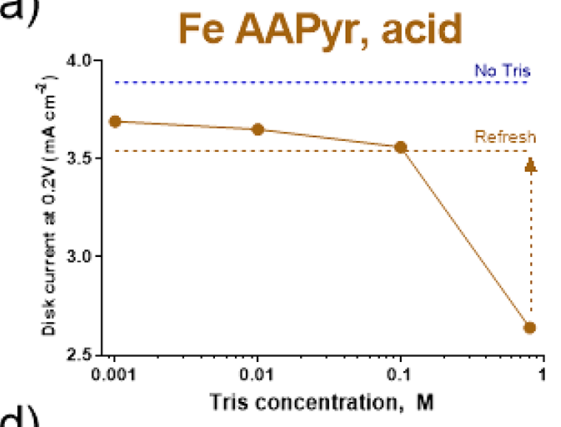

d)
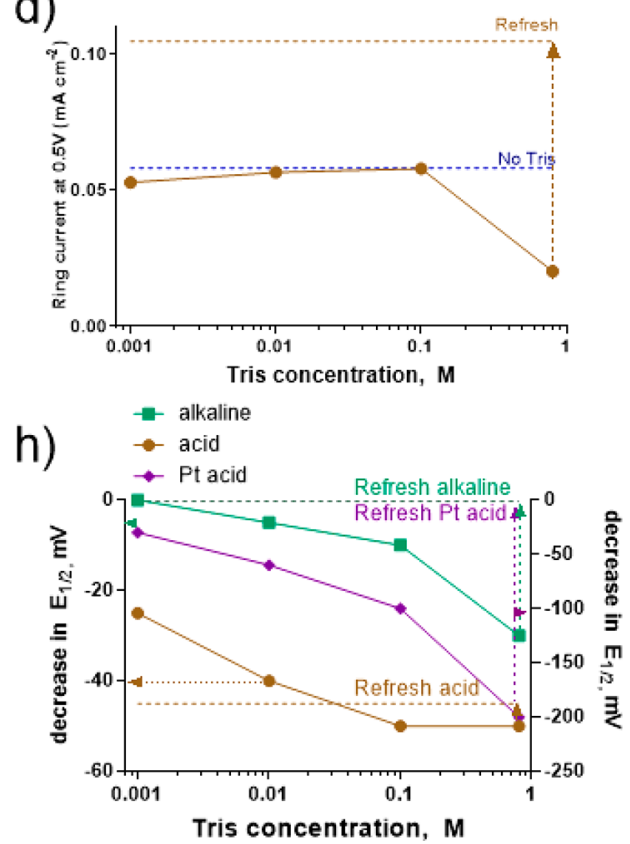

b)
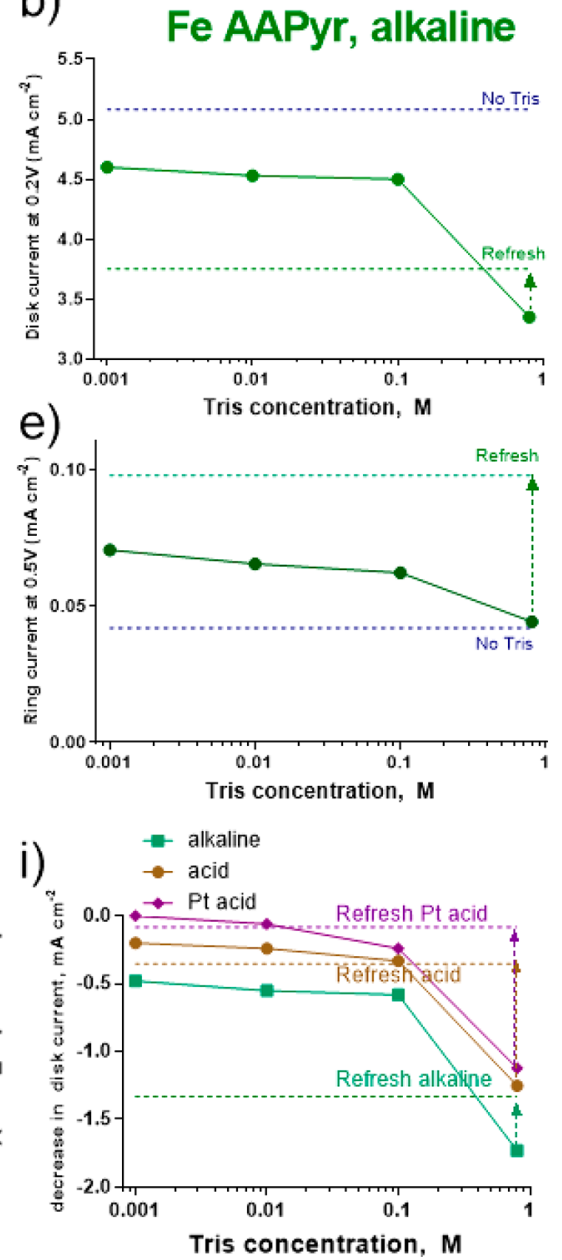

c)
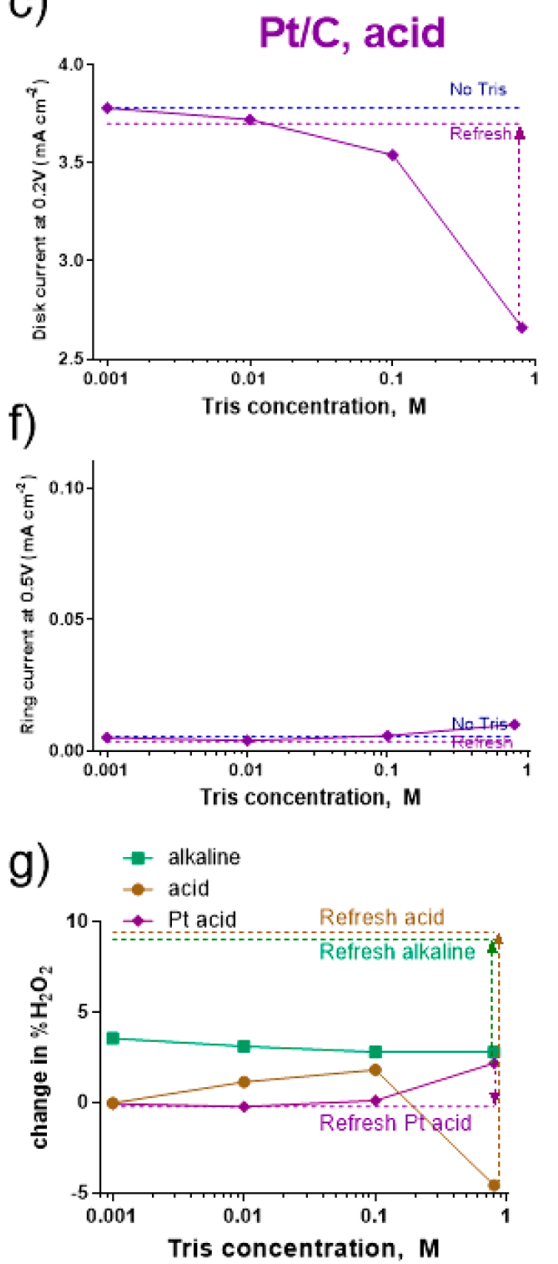

Figure 3. Electrochemical parameters extracted from LSV. Disk and ring current density for FeAApyr in (a, d) acid, (b, e) FeAApyr in alkaline, and (c, f) $10 \% \mathrm{Pt} / \mathrm{C}$ in acid. Changes in parameters for three systems: (h) decrease in half-wave potential; (i) decrease in disk current density; and (g) change in $\%$ generated $\mathrm{H}_{2} \mathrm{O}_{2}$.

acidic electrolyte, there is a $45 \mathrm{mV}$ downshift in the half-wave potential at $0.1 \mathrm{M}$ of Tris, but no further decrease in half-wave potential is observed when Tris concentrations were increased up to $0.8 \mathrm{M}$. The rate of change in half-wave potential is quite different in alkaline electrolyte (Figure $3 \mathrm{~h}$ ), where 0.1 and 0.8 $\mathrm{M}$ Tris induce much smaller shift in half-wave potential of only 15 and $35 \mathrm{mV}$, respectively. The rate of decrease in the disk limiting current density is very similar for both acidic and alkaline solutions (Figure 3i) with maximum decrease observed for $0.8 \mathrm{M}$ Tris concentration induced by decreased solubility of oxygen (as observed on $\mathrm{Pt} / \mathrm{C}$ ), especially at high concentrations of an inhibiting agent. The larger effect on disk current density in alkaline media versus acidic even at low concentrations of Tris points to the higher solubility of Tris in alkaline electrolyte.

After washing the working electrode with deionized water, a very small increase in $E_{1 / 2}(\sim 5 \mathrm{mV})$ was registered in the acidic electrolyte, which indicates the irreversible inhibition of active sites participating in the ORR by the Tris added (Figure $3 \mathrm{~h}$ ). At the same time, the half-wave potential is almost fully recovered in alkaline media. However, in both cases, the diffusion limiting current for the "recovered" catalysts are lower than observed before Tris-addition. This is easily explained by the higher peroxide yield (Figures $2 \mathrm{~b}, \mathrm{~d}$ and $3 \mathrm{~g}$ ) that induces a decrease of the overall number of electrons exchanged during the reaction and, therefore, a diminished diffusion limiting current. Differences in peroxide yield arise from the addition of Tris, which results in drastically different behavior due to a different mechanism of oxygen reduction, particularly in the first step of oxygen reduction to hydrogen peroxide. A significant difference between ORR in acidic and alkaline media is based on an innersphere and outer-sphere mechanism. In alkaline media, surface hydroxyl groups promote surface-independent outer-sphere electron transfer, whereas in acidic media immediate involvement of active sites such as metal coordinated to nitrogen in inner-sphere electron transfer is of direct relevance. ${ }^{39-41}$

In acidic media, Tris is present in its protonated form TrisH $\left(\mathrm{p} K_{\mathrm{a}}=8.07\right)$; the addition of low concentrations of Tris, i.e., between $1 \mathrm{mM}$ and $0.1 \mathrm{M}$ results in a similar decrease in a ring current due to TrisH inhibiting hydrogen peroxide producing sites. At large Tris concentration of $0.8 \mathrm{M}$, there is a significant decrease in hydrogen peroxide production due to a combination of decreased oxygen solubility and inhibition of $\mathrm{H}_{2} \mathrm{O}_{2}$ producing sites by bound TrisH. However, after washing the working electrode, the ring current density increased from $0.02 \mathrm{~mA} \mathrm{~cm}^{-2}$ to $0.10 \mathrm{~mA} \mathrm{~cm}{ }^{-2}$ at $0.5 \mathrm{~V}$ vs RHE (Figure $3 \mathrm{~d}$ ). This phenomenon of hydrogen peroxide recovery after catalyst washing is a clear indication of Tris reversible weak binding to the active centers participating in the first step of ORR mechanism. Moreover, DFT calculations show that the proton 

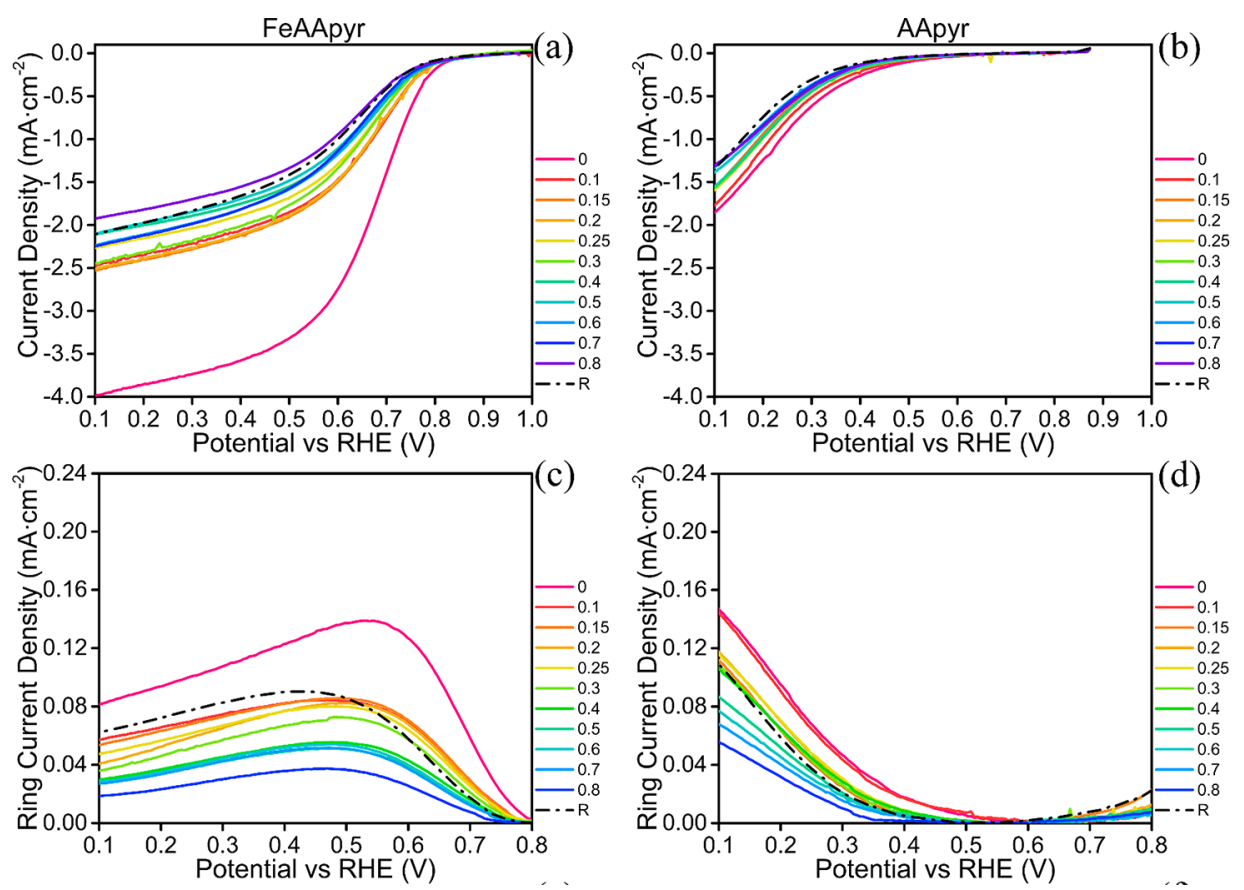

Figure 4. LSV data of (a, c) Fe-AApyr and (b, d) AApyr materials. (a, b) Disk current density and (c, d) ring current density.

from TrisH is being transferred to pyridinic nitrogen due to the lower $\mathrm{pK}$ value of the pyridinic nitrogen (6.5 for pyridinic nitrogen and 8.06 for Tris molecule). The interaction energy between protonated pyridinic nitrogen, and Tris molecule is only $-0.26 \mathrm{eV}$. In previous studies,protonation of pyridine has shown to inhibit oxygen reduction reaction. ${ }^{8}$ Pyridinic nitrogen has shown to catalyze the second step of hydrogen peroxide reduction to water. This implies that after the catalyst washing, TrisH is removed, but pyridinic nitrogen remains in its protonated form, therefore, explaining the lower half-wave potential and higher hydrogen peroxide yield of the "recovered" catalysts compared to their "fresh" counterpart (Figures 2a, b). Furthermore, on the basis of the DFT results in Table 1, TrisH can be more easily removed from protonated pyridinic nitrogen, pyrrolic nitrogen, and graphitic nitrogen, while $\mathrm{Fe}-$ $\mathrm{Nx}$ sites may be irreversibly blocked by TrisH.

In alkaline media, Tris is present in its deprotonated form. The interaction energy between the $\mathrm{Fe}-\mathrm{N}_{4}$ site and Tris was determined to be $-0.25 \mathrm{eV}$, significantly smaller than that of TrisH (Figure S1, Table S1). DFT has shown competitive binding of Tris and oxygen to pyridinic nitrogen. Enhanced hydrogen peroxide generation at all concentrations of Tris is observed (Figure 3g). This can be attributed to the presence of Tris bound to pyridinic nitrogen within the outer Helmholtz sphere of the catalyst surface, which enhances the first step of oxygen reduction to peroxide. ${ }^{40,41}$

In conclusion, in acidic electrolyte, TrisH interacts strongly with active sites that catalyze both complete and partial reduction of oxygen, and, therefore, it should inhibit both steps of ORR. As observed in the experiment performed in an acidic electrolyte, the addition of TrisH results in a decrease of both half-wave potential and the ring current confirming direct blocking of active sites for both complete and partial oxygen reduction by TrisH. In alkaline electrolyte, Tris can only competitively bind to pyridinic nitrogen which has been shown to catalyze the reduction of hydrogen peroxide to water. ${ }^{7}$ The half-wave potential recorded in alkaline media is changed very slightly by the addition of Tris. However, there is an enhancement of hydrogen peroxide production, observed experimentally when Tris is added to the alkaline electrolyte. One explanation could be that specific adsorption of Tris leads to selective blocking of pyridinic nitrogen moieties, which act as catalytic sites for hydrogen peroxide reduction to water. This effect of selective site blocking can be synergistic to a local increase of $-\mathrm{OH}$ groups in proximity of Tris-bound to pyridinic nitrogen surface groups, which can be beneficial to oxygen reduction to hydrogen peroxide in alkaline electrolite. In acidic electrolyte TrisH affects both partial oxygen reduction to peroxide and complete oxygen reduction to water, as evidenced by the observation that both half-wave potential and the peroxide yield decrease for Tris concentrations exceeding $0.1 \mathrm{M}$. In contrast, in alkaline electrolyte Tris promotes the first step of reaction of producing hydrogen peroxide and inhibits the second step of $\mathrm{H}_{2} \mathrm{O}_{2}$ reduction to water. This alternate effect on selectivity allows one to use Tris as a homogeneous, electrolyte-based "titration agent" to selectively study kinetics of individual steprs of ORR on various catalytically active moeties prsent in $\mathrm{M}-\mathrm{N}-\mathrm{C}$ catalysts.

To address the role of metal on oxygen reduction mechanism we have tested inhibition of the electrocatalytic activity of FeAAPyr and AAPyr by Rotating Ring Disk Electrode (RRDE) method in the $1 \mathrm{M} \mathrm{HClO}_{4}$ acidic electrolyte with the addition of Tris as the inhibiting agent (Figure 4). The electrochemical information derived from linear sweep voltammograms (LSVs) as a function of Tris concentration is presented in Figure S2. Like the experiments reported in Figure 2, in the case of FeAApyr, the shift to lower values in half-wave potential with increasing concentration of Tris was observed (Figure S2 a). When $0.8 \mathrm{M}$ Tris is added to the acidic electrolyte, similar shift of $50 \mathrm{mV}$ is observed in both $0.5 \mathrm{M} \mathrm{H}_{2} \mathrm{SO}_{4}$ and $1 \mathrm{M} \mathrm{HClO}_{4}$. Very insignificant recovery in half-wave potential indicates the irreversible inhibition of active sites participating in the ORR. After this initial decrease in ORR activity upon addition of 0.1 $M$ Tris, increasing the Tris concentration resulted in the 
insignificant decrease of the limiting current density (Figure 5a).

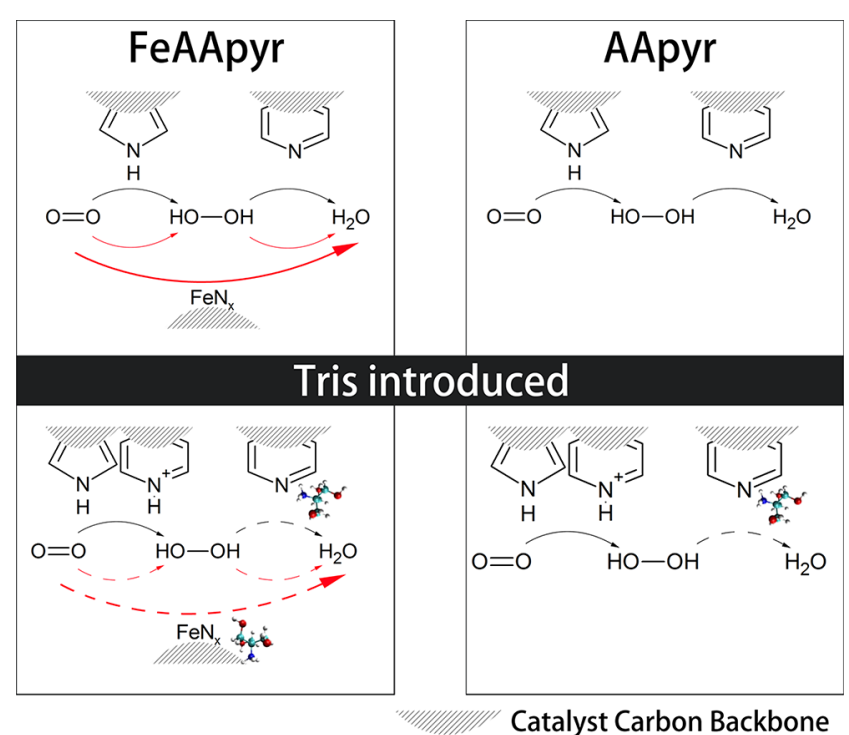

Figure 5. Schematic diagram of ORR mechanism changes before and after Tris introduced into the solution. Catalytically active surface moieties are depicted schematically with no intention of implying any special positioning of distribution in the actual material. "Dashed" lines suppressed reactions subject to reversible or irreversible inhibition.

As can be seen in Figure 4c, the ring current density decreased with the increase of the Tris concentration from 0 to 0.8 M. Partial recovery of electrochemical production of $\mathrm{H}_{2} \mathrm{O}_{2}$ after washing the catalyst is an indication of weak adsorption of Tris on hydrogen peroxide -producing active sites.

The similar set of experiments were performed with metalfree (AAPyr) electrocatalyst to discriminate further the active sites participating in ORR. It was found that upon exposure of metal-free catalyst (AAPyr) to Tris, both half-wave and disk current densities gradually decrease (Figure $4 b, d$ ). In general, the overall half-wave potential is not recovered after washing, in contrast to ring current densities, which approaches the values obtained in the Tris-free electrolyte.

In both $\mathrm{Fe}-\mathrm{N}-\mathrm{C}$ and $\mathrm{N}-\mathrm{C}$ materials, pyridinic nitrogen and hydrogenated nitrogen (pyrrolic and hydrogenated pyridine) are part of the $2 \times 2 \mathrm{e}^{-}$mechanism, in which hydrogenated nitrogen reduces oxygen to $\mathrm{H}_{2} \mathrm{O}_{2}$, while pyridinic nitrogen reduces $\mathrm{H}_{2} \mathrm{O}_{2}$ to water. Comparison between inhibition and recovery of ORR performance in metal-free and metalcontaining electrocatalysts shows that the moieties that reduce oxygen directly to water $\left(\mathrm{Fe}-\mathrm{N}_{x}\right)$ have a higher affinity to TrisH compared to the active centers reducing oxygen to hydrogen peroxide (pyridinic and hydrogenated nitrogen). The metal-containing catalyst contains sites that have stronger binding of TrisH as established by the drastic decrease in the half-wave potential. TrisH also adsorbs onto both hydrogenated nitrogen and pyridinic nitrogen more strongly than $\mathrm{O}_{2}$, inhibiting, thus, both the first and the second step of the ORR. However, upon Tris removal by washing, protonation of pyridine results in the creation of sites that reduce oxygen to hydrogen peroxide, which manifests itself as a recovery in the hydrogen peroxide yield. In metal-containing electrocatalysts, $\mathrm{Fe}-\mathrm{N}_{x}$ moieties are the most active toward the full reduction of oxygen to water via either $4 \mathrm{e}^{-}$or $2 \times 2 \mathrm{e}^{-}$mechanism. At the same time, TrisH binds strongly and irreversibly to these sites, more strongly than $\mathrm{O}_{2}$, and inhibits the oxygen reduction reaction. After removal of the TrisH by washing, most of the sites are protonated and contribute to the production of hydrogen peroxide.

To summarize, in Figure 5, we suggest the possible mechanism of oxygen reduction and changes in surface chemistry that occur when inhibitor Tris is introduced into the acidic solution. In this diagram, we show that the $\mathrm{Fe}-\mathrm{N}_{x}$ centers are being irreversibly blocked by TrisH resulting in slower direct $4 \mathrm{e}^{-}$oxygen reduction to water. At the same time protonation of pyridinic nitrogen results in the enhanced kinetics of the first step of reduction of oxygen to hydrogen peroxide.

\section{CONCLUSION}

Poisoning effect of Tris(hydroxymethyl)aminomethane (Tris) on the electrocatalytic activity of $\mathrm{M}-\mathrm{N}-\mathrm{C}$ toward ORR in the acidic and alkaline environment was comprehensively studied experimentally and computationally. The experiments in acidic electrolyte also allowed to analyze the role of metal in $\mathrm{M}-\mathrm{N}-\mathrm{C}$ by studying the effect of the inhibitor on the electrochemical behavior of metal free and metal-containing electrocatalyst. The introduction of Tris into the electrolyte inhibits iron-nitrogen and nitrogen-carbon active sites. Rotating ring disk electrode experiments and density functional theory calculations show that the $\mathrm{Fe}-\mathrm{N}_{x}$ sites could be irreversibly poisoned while $\mathrm{N}-\mathrm{C}$ sites can be recovered by the removal of Tris. Density functional theory calculations also show that the protonation of pyridinic nitrogen could contribute to the recovery of the hydrogen peroxide generation in both metal-free and metal containing electrocatalysts in acidic media. As Tris is a commonly used chemical in electrochemistry research, the described phenomena reveal new avenues for mechanistic studies of PGM-free oxygen reduction catalysts.

\section{ASSOCIATED CONTENT}

\section{S Supporting Information}

The Supporting Information is available free of charge on the ACS Publications website at DOI: 10.1021/acsaem.8b00020.

Figures S1 and S2 and Table S1 (PDF)

\section{AUTHOR INFORMATION}

\section{Corresponding Authors}

*E-mail: kartyush@unm.edu.

*E-mail: plamen@unm.edu.

ORCID $\odot$

K. Artyushkova: 0000-0002-2611-0422

I. Matanovic: 0000-0002-9191-8620

P. Atanassov: 0000-0003-2996-472X

\section{Author Contributions}

The manuscript was written through contributions of all authors. S.R.-C. discovered the inhibitor effect; Y.Ch., S.R.-C., C.S., and T.A. were primarily responsible for the electrochemical evaluation; S.R.-C., A.S., and T.A. for synthesis; I.M. for DFT calculations; Y.Ch., T.A., K.A., and P.A. for planning, analysis, and writing. All authors have given approval to the final version of the manuscript.

Notes

The authors declare no competing financial interest. 


\section{ACKNOWLEDGMENTS}

We thankfully acknowledge the computational resources for this project from the Tri-Lab computing resources of LANL, operated by Los Alamos National Security, LLC, for the National Nuclear Security Administration of U.S. Department of Energy (Contract DE-AC52-06NA25396), computational resources of NERSC, a DOE Office of Science User Facility supported by the Office of Science of the U.S. Department of Energy under Contract No. DE-AC02-05CH11231, and CNMS, which is a DOE Office of Science User Facility. VASP license was provided by Theoretical division, LANL. This paper has been designated LA-UR-17-30624.

\section{REFERENCES}

(1) Zhang, W.; Lai, W.; Cao, R. Energy-Related Small Molecule Activation Reactions: Oxygen Reduction and Hydrogen and Oxygen Evolution Reactions Catalyzed by Porphyrin- and Corrole-Based Systems. Chem. Rev. 2017, 117 (4), 3717-3797.

(2) Lai, Q.; Zheng, L.; Liang, Y.; He, J.; Zhao, J.; Chen, J. MetalOrganic-Framework-Derived Fe-N/C Electrocatalyst with Five-Coordinated Fe-Nx Sites for Advanced Oxygen Reduction in Acid Media. ACS Catal. 2017, 7 (3), 1655-1663.

(3) Chen, Y.; Gokhale, R.; Serov, A.; Artyushkova, K.; Atanassov, P. Novel highly active and selective $\mathrm{Fe}-\mathrm{N}-\mathrm{C}$ oxygen reduction electrocatalysts derived from in-situ polymerization pyrolysis. Nano Energy 2017, 38, 201-209.

(4) Chung, H. T.; Cullen, D. A.; Higgins, D.; Sneed, B. T.; Holby, E. F.; More, K. L.; Zelenay, P. Direct atomic-level insight into the active sites of a high-performance PGM-free ORR catalyst. Science 2017, 357 (6350), 479-484.

(5) Sa, Y. J.; Seo, D.-J.; Woo, J.; Lim, J. T.; Cheon, J. Y.; Yang, S. Y.; Lee, J. M.; Kang, D.; Shin, T. J.; Shin, H. S.; Jeong, H. Y.; Kim, C. S.; Kim, M. G.; Kim, T.-Y.; Joo, S. H. A General Approach to Preferential Formation of Active $\mathrm{Fe}-\mathrm{Nx}$ Sites in $\mathrm{Fe}-\mathrm{N} / \mathrm{C}$ Electrocatalysts for Efficient Oxygen Reduction Reaction. J. Am. Chem. Soc. 2016, 138 (45), 15046-15056.

(6) Jia, Q.; Ramaswamy, N.; Tylus, U.; Strickland, K.; Li, J.; Serov, A.; Artyushkova, K.; Atanassov, P.; Anibal, J.; Gumeci, C.; Barton, S. C.; Sougrati, M.-T.; Jaouen, F.; Halevi, B.; Mukerjee, S. Spectroscopic insights into the nature of active sites in iron-nitrogen-carbon electrocatalysts for oxygen reduction in acid. Nano Energy 2016, 29, $65-82$.

(7) Artyushkova, K.; Serov, A.; Rojas-Carbonell, S.; Atanassov, P. Chemistry of multitudinous active sites for oxygen reduction reaction in transition metal-nitrogen-carbon electrocatalysts. J. Phys. Chem. C 2015, 119 (46), 25917-25928.

(8) Mamtani, K.; Jain, D.; Zemlyanov, D.; Celik, G.; Luthman, J.; Renkes, G.; Co, A. C.; Ozkan, U. S. Probing the Oxygen Reduction Reaction Active Sites over Nitrogen-Doped Carbon Nanostructures $(\mathrm{CNx})$ in Acidic Media Using Phosphate Anion. ACS Catal. 2016, 6 (10), 7249-7259.

(9) Zitolo, A.; Goellner, V.; Armel, V.; Sougrati, M.-T.; Mineva, T.; Stievano, L.; Fonda, E.; Jaouen, F. Identification of catalytic sites for oxygen reduction in iron- and nitrogen-doped graphene materials. Nat. Mater. 2015, 14 (9), 937-942.

(10) von Deak, D.; Singh, D.; Biddinger, E. J.; King, J. C.; Bayram, B.; Miller, J. T.; Ozkan, U. S. Investigation of sulfur poisoning of $\mathrm{CNx}$ oxygen reduction catalysts for PEM fuel cells. J. Catal. 2012, 285 (1), $145-151$.

(11) Singh, D.; Mamtani, K.; Bruening, C. R.; Miller, J. T.; Ozkan, U. S. Use of H2S to Probe the Active Sites in FeNC Catalysts for the Oxygen Reduction Reaction (ORR) in Acidic Media. ACS Catal. 2014, 4 (10), 3454-3462.

(12) von Deak, D.; Singh, D.; King, J. C.; Ozkan, U. S. Use of carbon monoxide and cyanide to probe the active sites on nitrogen-doped carbon catalysts for oxygen reduction. Appl. Catal., B 2012, 113, 126133.
(13) Tylus, U.; Jia, Q.; Hafiz, H.; Allen, R. J.; Barbiellini, B.; Bansil, A.; Mukerjee, $S$. Engendering anion immunity in oxygen consuming cathodes based on Fe-Nx electrocatalysts: Spectroscopic and electrochemical advanced characterizations. Appl. Catal., B 2016, 198, 318324.

(14) Santoro, C.; Serov, A.; Villarrubia, C. W. N.; Stariha, S.; Babanova, S.; Artyushkova, K.; Schuler, A. J.; Atanassov, P. High catalytic activity and pollutants resistivity using Fe-AAPyr cathode catalyst for microbial fuel cell application. Sci. Rep. 2015, 5, 16596.

(15) Santoro, C.; Serov, A.; Stariha, L.; Kodali, M.; Gordon, J.; Babanova, S.; Bretschger, O.; Artyushkova, K.; Atanassov, P. Iron based catalysts from novel low-cost organic precursors for enhanced oxygen reduction reaction in neutral media microbial fuel cells. Energy Environ. Sci. 2016, 9 (7), 2346-2353.

(16) Zhang, C.; An, B.; Yang, L.; Wu, B.; Shi, W.; Wang, Y.-C.; Long, L.-S.; Wang, C.; Lin, W. Sulfur-doping achieves efficient oxygen reduction in pyrolyzed zeolitic imidazolate frameworks. J. Mater. Chem. A 2016, 4 (12), 4457-4463.

(17) Kneebone, J. L.; Daifuku, S. L.; Kehl, J. A.; Wu, G.; Chung, H. T.; Hu, M. Y.; Alp, E. E.; More, K. L.; Zelenay, P.; Holby, E. F.; Neidig, M. L. A Combined Probe-Molecule, Mössbauer, Nuclear Resonance Vibrational Spectroscopy, and Density Functional Theory Approach for Evaluation of Potential Iron Active Sites in an Oxygen Reduction Reaction Catalys. J. Phys. Chem. C 2017, 121 (30), 16283-16290.

(18) Malko, D.; Kucernak, A.; Lopes, T. Performance of Fe-N/C Oxygen Reduction Electrocatalysts toward $\mathrm{NO} 2-, \mathrm{NO}$, and $\mathrm{NH} 2 \mathrm{OH}$ Electroreduction: From Fundamental Insights into the Active Center to a New Method for Environmental Nitrite Destruction. J. Am. Chem. Soc. 2016, 138 (49), 16056-16068.

(19) Janarthanan, R.; Serov, A.; Pilli, S. K.; Gamarra, D. A.; Atanassov, P.; Hibbs, M. R.; Herring, A. M. Direct Methanol Anion Exchange Membrane Fuel Cell with a Non-Platinum Group Metal Cathode based on Iron-Aminoantipyrine Catalyst. Electrochim. Acta 2015, 175 (Suppl C), 202-208.

(20) Sebastián, D.; Baglio, V.; Aricò, A. S.; Serov, A.; Atanassov, P. Performance analysis of a non-platinum group metal catalyst based on iron-aminoantipyrine for direct methanol fuel cells. Appl. Catal., B 2016, 182 (Suppl C), 297-305.

(21) Serov, A.; Robson, M. H.; Halevi, B.; Artyushkova, K.; Atanassov, P. Highly active and durable templated non-PGM cathode catalysts derived from iron and aminoantipyrine. Electrochem. Commun. 2012, 22 (Suppl C), 53-56.

(22) Robson, M. H.; Serov, A.; Artyushkova, K.; Atanassov, P. A mechanistic study of 4-aminoantipyrine and iron derived non-platinum group metal catalyst on the oxygen reduction reaction. Electrochim. Acta 2013, 90, 656-665.

(23) Rojas-Carbonell, S.; Babanova, S.; Serov, A.; Artyushkova, K.; Workman, M. J.; Santoro, C.; Mirabal, A.; Calabrese Barton, S.; Atanassov, P. Integration of Platinum Group Metal-Free Catalysts and Bilirubin Oxidase into a Hybrid Material for Oxygen Reduction: Interplay of Chemistry and Morphology. ChemSusChem 2017, 10 (7), 1534-1542.

(24) Rojas-Carbonell, S.; Santoro, C.; Serov, A.; Atanassov, P. Transition metal-nitrogen-carbon catalysts for oxygen reduction reaction in neutral electrolyte. Electrochem. Commun. 2017, 75, 38-42.

(25) Blöchl, P. E. Projector augmented-wave method. Phys. Rev. B: Condens. Matter Mater. Phys. 1994, 50 (24), 17953-17979.

(26) Kresse, G.; Joubert, D. From ultrasoft pseudopotentials to the projector augmented-wave method. Phys. Rev. B: Condens. Matter Mater. Phys. 1999, 59 (3), 1758-1775.

(27) Perdew, J. P.; Burke, K.; Ernzerhof, M. Generalized Gradient Approximation Made Simple. Phys. Rev. Lett. 1996, 77 (18), 38653868.

(28) Perdew, J. P.; Burke, K.; Ernzerhof, M. Generalized Gradient Approximation Made Simple. Phys. Rev. Lett. 1996, 77, 3865. Perdew, J. P.; Burke, K.; Ernzerhof, M. Generalized Gradient Approximation Made Simple [Phys. Rev. Lett. 77, 3865 (1996)]. Phys. Rev. Lett. 1997, 78 (7), 1396-1396. 
(29) Kresse, G.; Furthmüller, J. Efficient iterative schemes for ab initio total-energy calculations using a plane-wave basis set. Phys. Rev. B: Condens. Matter Mater. Phys. 1996, 54 (16), 11169-11186.

(30) Kresse, G.; Hafner, J. Ab initio molecular-dynamics simulation of the liquid-metal-amorphous-semiconductor transition in germanium. Phys. Rev. B: Condens. Matter Mater. Phys. 1994, 49 (20), 1425114269.

(31) Blöchl, P. E.; Jepsen, O.; Andersen, O. K. Improved tetrahedron method for Brillouin-zone integrations. Phys. Rev. B: Condens. Matter Mater. Phys. 1994, 49 (23), 16223-16233.

(32) Monkhorst, H. J.; Pack, J. D. Special points for Brillouin-zone integrations. Phys. Rev. B 1976, 13 (12), 5188-5192.

(33) Kattel, S.; Atanassov, P.; Kiefer, B. Stability, Electronic and Magnetic Properties of In-Plane Defects in Graphene: A FirstPrinciples Study. J. Phys. Chem. C 2012, 116 (14), 8161-8166.

(34) Sebastian, D.; Serov, A.; Matanovic, I.; Artyushkova, K.; Atanassov, P.; Arico, A. S.; Baglio, V. Insights on the extraordinary tolerance to alcohols of Fe-N-C cathode catalysts in highly performing direct alcohol fuel cells. Nano Energy 2017, 34, 195-204.

(35) Artyushkova, K.; Matanovic, I.; Halevi, B.; Atanassov, P. Oxygen Binding to Active Sites of $\mathrm{Fe}-\mathrm{N}-\mathrm{C}$ ORR Electrocatalysts Observed by Ambient-Pressure XPS. J. Phys. Chem. C 2017, 121 (5), 28362843.

(36) Šljivančanin, Ž.; Hammer, B. Oxygen dissociation at closepacked Pt terraces, Pt steps, and Ag-covered Pt steps studied with density functional theory. Surf. Sci. 2002, 515 (1), 235-244.

(37) Hyman, M. P.; Medlin, J. W. Theoretical Study of the Adsorption and Dissociation of Oxygen on $\mathrm{Pt}(111)$ in the Presence of Homogeneous Electric Fields. J. Phys. Chem. B 2005, 109 (13), 63046310.

(38) Li, R.; Li, H.; Liu, J. First principles study of O2 dissociation on $\mathrm{Pt}$ (111) surface: Stepwise mechanism. Int. J. Quantum Chem. 2016, 116 (12), 908-914.

(39) Gokhale, R.; Tsui, L. K.; Roach, K.; Chen, Y.; Hossen, M. M.; Artyushkova, K.; Garzon, F.; Atanassov, P., Hydrothermal Synthesis of Platinum-Group-Metal-Free Catalysts: Structural Elucidation and Oxygen Reduction Catalysis. ChemElectroChem 2017, 10.1002/ celc.201700949.

(40) Rojas-Carbonell, S.; Artyushkova, K.; Serov, A.; Santoro, C.; Matanovic, I.; Atanassov, P. Effect of $\mathrm{pH}$ on the Activity of Platinum Group Metal-Free Catalysts in Oxygen Reduction Reaction. ACS Catal. 2018, 8, 3041-3053.

(41) Ramaswamy, N.; Mukerjee, S. Influence of Inner- and OuterSphere Electron Transfer Mechanisms during Electrocatalysis of Oxygen Reduction in Alkaline Media. J. Phys. Chem. C 2011, 115 (36), 18015-18026. 